\title{
Two predicted models based on ceRNAs and immune cells in lung adenocarcinoma
}

\author{
Miaomiao Zhang ${ }^{\text {Equal first author, } 1}$, Peiyan Zheng $\square$ Peiyan $\square^{\text {Equal first author, } 2}$, Yuan Wang ${ }^{\text {Corresp., } 1}$, Baoqing Sun(Baoqing) ${ }^{\text {Corresp. } 2}$ \\ ${ }^{1}$ The First Affiliated Hospital of Guangzhou Medical University, Guangzhou Institute of Respiratory Diseases, State Key Laboratory of Respiratory Disease, \\ GuangZhou, China \\ 2 Department of Allergy and Clinical Immunology, Guangzhou Institute of Respiratory health, State Key Laboratory of Respiratory Disease, National \\ Clinical Research Center of Respiratory Disease, First Affiliated Hospital of Guangzhou Medical University, State Key Laboratory of Respiratory Disease, \\ Guangzhou, china \\ Corresponding Authors: Yuan Wang, Baoqing Sun(Baoqing) \\ Email address: YuanWangxz1992@126.com, sunbaoqing@vip.163.com
}

Background It is well accepted that both competitive endogenous RNAs (ceRNAs) and immune microenvironment exert crucial roles in the tumor prognosis. The present study aimed to find prognostic ceRNAs and immune cells in lung adenocarcinoma (LUAD).

Materials and Methods More specifically, we explored the associations of crucial ceRNAs with the immune microenvironment. The cancer genome atlas (TCGA) database was employed to obtain expression profiles of ceRNAs and clinical data. CIBERSORT was utilized to quantify the proportion of 22 immune cells in LUAD. Results We constructed two cox regression models based on crucial ceRNAs and immune cells to predict prognosis in LUAD. Subsequently, seven ceRNAs and seven immune cells were involved in prognostic models. We validated both predicted models via an independent cohort GSE72094. Interestingly, both predicted models proved that the longer patients were smoking, the higher risk scores would be obtained. We further investigated the relationships between seven genes and immune/stromal scores via the ESTIMATE algorithm. The results indicated that CDC14A and H1F0 expression were significantly related to stromal scores/Immune scores in LUAD. Moreover, based on the result of the ceRNA model, singlesample gene set enrichment analysis (ssGSEA) suggested that differences in immune status were evident between high- and low-risk groups. 


\section{Two predicted models based on ceRNAs and immune cells in 2 lung adenocarnoma}

3 Miaomiao Zhang ${ }^{1}$,PeiYan Zheng ${ }^{2}$,Yuan Wang ${ }^{1}$,BaoQing Sun $^{2}$

$4 \quad{ }^{1}$ The First Affiliated Hospital of Guangzhou Medical University, Guangzhou Institute of

5 Respiratory Diseases, State Key Laboratory of Respiratory Disease, 151 Yanjiang

6 Road,Guangzhou 510120, China

$7 \quad{ }^{2}$ Department of Allergy and Clinical Immunology, Guangzhou Institute of Respiratory health 8 , State Key Laboratory of Respiratory Disease, National Clinical Research Center of Respiratory 9 Disease, First Affiliated Hospital of Guangzhou Medical University, Guangzhou, China

11 Correspondence Author:

12 Yuan Wang, YuanWangxz1992@126.com

13 SunBao Qing,Sunbaoqing@vip.163.com

15 Keywords lung adenocarcinoma, immune microenvironment, prognosis,ceRNA (competitive 16 endogenous RNAs). 
Running Title: TWO PREDICTED MODELS in LUAD

\section{Abstract}

Background It is well accepted that both competitive endogenous RNAs (ceRNAs) and immune microenvironment exert crucial roles in the tumor prognosis. The present study aimed to find prognostic ceRNAs and immune cells in lung adenocarcinoma (LUAD). Materials and Methods More specifically, we explored the associations of crucial ceRNAs with the immune microenvironment. The cancer genome atlas (TCGA) database was employed to obtain expression profiles of ceRNAs and clinical data. CIBERSORT was utilized to quantify the proportion of 22 immune cells in LUAD. Results We constructed two cox regression models based on crucial ceRNAs and immune cells to predict prognosis in LUAD. Subsequently, seven ceRNAs and seven immune cells were involved in prognostic models. We validated both predicted models via an independent cohort GSE72094. As expected, both predicted models proved that the longer smoking time was related to the higher risk scores among cigarette smokers. We further investigated the relationships between seven genes and immune/stromal scores via the ESTIMATE algorithm. The results indicated that $\mathrm{CDC} 14 \mathrm{~A}$ and $\mathrm{H} 1 \mathrm{~F} 0$ expression were significantly related to stromal scores/Immune scores in LUAD. Moreover, based on the result of the ceRNA model, single-sample gene set enrichment analysis (ssGSEA) suggested that differences in immune status were evident between high- and low-risk groups. 


\section{Introduction}

37 Lung cancer is considered as the most common cause of survival-associated cancer globally(Bray

38 et al. 2018). Besides, lung adenocarcinoma (LUAD) approximately accounts for $50 \%$ of all lung

39 cancer types (Imielinski et al. 2012). Although the improvement of diagnosis and the

40 comprehensive treatment in the lung cancer has been made, the 5-year overall survival rate for

41 lung cancer is still approximately 18\%, mainly due to invisible early symptoms (Siegel et al. 2018).

42 Thus, it is of great necessity to identify sensitive and accurate biomarkers in the early stage.

43 Competitive endogenous RNAs (ceRNAs), consisting of a group of regulatory RNA molecules,

44 bind with the specific miRNAs and then affect the protein level (Kartha \& Subramanian 2014;

45 Phelps et al. 2016; Shukla et al. 2011). Previous studies have shown that ceRNA regulatory

46 networks play essential roles in tumor progression and migration (Bartel 2009; Qi et al. 2015;

47 Sanchez-Mejias \& Tay 2015; Tay et al. 2014). Besides, increasing studies have demonstrated that

48 immune microenvironment (TIME) was also associated with the prognosis in various kinds of

49 tumors, including LUAD (Lavin et al. 2017; Liu et al. 2020a). Stromal cells and immune cells

50 constitute the central part of TIME. Stromal cells participate in the process of tumorigenesis and

51 metastasis and are generally conducive to the movement of cancer cells (Ni et al. 2018).

52 Additionally, various studies revealed that immune cells exhibit large effects on clinical outcomes

53 in many cancers. Donnem et al. summarized that different types of immune cells affected the

54 clinical stage and prognosis in non-small lung cancer (Donnem et al. 2016; Liu et al. 2020b). As a

55 result, to further investigate potential biomarkers based on immune cells and ceRNAs in LUAD, 
56 we constructed Cox proportional hazards models and predicted nomograms.

57 However, the mechanisms of ceRNAs in the tumor microenvironment still remain unclear in

58 LUAD. It is well accepted that cancer stem cells (CSCs) are tumorigenic cells with self-renewal

59 ability. Besides, CSCs promote tumor immune to escape via expressing a variety of immune

60 factors (Bruttel \& Wischhusen 2014). In the current study, RNA stem scores and DNA stem scores

61 were applied to assess tumor stem cell content (Malta et al. 2018). ESTIMATE algorithm was

62 utilized to evaluate the stromal and immune cells in every sample (Yoshihara et al. 2013). Thus,

63 we explored the correlation between crucial prognostic genes with RNAss/DNAss scores and

64 stromal/immune scores. All in all, in this study, we comprehensively analyze the associations

65 between crucial ceRNAs and immune infiltration landscape, crucial ceRNAs and tumor stem cells,

66 which may offer an important perspective for early diagnosis and treatment of LUAD.

\section{Materials and Methods}

68 Identification of differentially expressed ceRNAs

69 In the present study, expression profiles of LUAD and the corresponding clinical information were

70 derived from the TCGA database, including lncRNA,miRNA, and mRNA. The R package

71 "DEseq2" was used to screen the differentially expressed mRNAs,miRNAs and lncRNAs

72 (DEmRNA, DEmiRNA and DElncRNA) between normal and tumor samples (Love et al.

73 2014).Specifically, a false discovery rate (FDR) $<0.05$ and $\log 2 \mid$ fold change $\mid>1$ were set as

74 cutoff criteria. 
75

76

77

ceRNA network construction

Both IncRNA-miRNA and miRNA-mRNA interactions were predicted using, miRcode and starBase(Chou et al. 2018; Jeggari et al. 2012; Li et al. 2014). DEmiRNAs, DEmRNAs, and DE lncRNAs, which presented a significant difference in hypergeometric distribution detection and correlation analysis $(\mathrm{P}<0.05)$, were selected to construct a lncRNA-miRNA-mRNA regulatory network and then was visualized by Cytoscape 3.80 (Shannon et al. 2003).

\section{Construction of nomogram based on key ceRNAs}

Univariate Cox analysis was employed to screen the survival-associated ceRNAs. Lasso regression analysis was applied to ensure that the Cox model was not overfitted. Eventually, the candidate ceRNAs were integrated to establish the multivariate cox regression models. Risk scores were described as following: risk score $=\left(\operatorname{Exp} \mathrm{RNA}^{*} \beta 1\right)+(\operatorname{Exp} \mathrm{RNA} 2 * \beta 2)+\ldots+(\operatorname{Exp} \mathrm{RNAn} *$ $\beta$ n). It is worth noting that Exp stands for the expression level, and $\beta$ denotes the regression coefficient. Based on the risk scores, patients with LUAD were divided into high-risk and low-risk groups. The corresponding Kaplan-Meier survival curves were carried out to reveal the overall survival (OS) within different groups. In addition, we also performed the ROC curve to evaluate the specificity and sensitivity of the model. Finally, according to the multivariate model result, we constructed a nomogram to predict patients' prognostic values. The calibration curve was applied to evaluate the accuracy of the nomogram.

\section{Construction of nomogram based on immune cells}


94 With a good deconvolution performance in gene expression profiles, CIBERSORT method could

95

96

97

98

99

100

101

102

103

104

105

106

107

108

109

110

111

112

113 estimate the proportion of particular cell types (Newman et al. 2015). In this study, CIBERSORT algorithm was utilized to estimate 22 tumour-infiltrating immune cells in LUAD by collating and calculating the genes expression in the tumor samples in the TCGA database. Samples with a CIBERSORT output of $\mathrm{p}<0.05$ presented that the predicted proportion of immune cells were correct.

The Wilcoxon test was conducted to examine the significant difference of immune cells between the tumor samples and healthy samples. We also performed multivariate Cox regression and produced the corresponding risk scores. Based on the multivariate Cox model results, we finally built a predicted nomogram. Similar to ceRNAs, patients were also stratified into high-risk and low-risk groups on the basis of the mean risk score. Subsequently, the prognostic model's sensitivity and specificity were examined by the ROC curve, and the calibration curve investigated the accuracy of the nomogram.

\section{Validation of prognostic equations with GEO}

To better elucidate the accuracy of both multivariate Cox models, an external validation dataset GSE72094 ( $\mathrm{n}=442)$ was applied in the present study. We selected this dataset because it contained most LUAD patients with clinical data in the Gene Expression Omnibus (GEO) database. Based on the median risk score generated from TCGA, we also classified patients with high- and lowrisk patients. K-M and ROC curves were applied to evaluate the prognostic efficacy of multivariate models in TCGA. 
114 Estimation of tumor stemness and microenvironment

115 Stemness scores of "RNAss" and "DNAss" referred to the result based on mRNA and DNA-

116 methylation respectively. The larger stemness scores were, the higher likelihood more stem cells

117 were infiltrating. ESTIMATE algorithm could speculate the level of stromal cell, immune cells

118 and tumor purity, respectively (Yoshihara et al. 2013). Correspondingly,

119 stromal/immune/Estimate scores were significantly associated with stromal cells, immune cells

120 and tumor purity, respectively. We utilized the "ESTIMATE" package to assess immune, stromal

121 scores, and the sum of both in individual patients. The higher scores, the higher proportion of

122 corresponding cells were. Additionally, the correlation of hub ceRNAs with above scores was

123 examined by Spearman analysis. The ssGSEA method could apply expressed traits of immune cell

124 population to individual tumor samples (Barbie et al. 2009). Based upon the results of ssGSEA,

125 we calculated infiltrating level of immune cells and immune-associated functions in LUAD

126 samples by using the "gsva" package (Hänzelmann et al. 2013). Furthermore, we compared the

127 differences in these immune data sets between the high-risk group and the low-risk group.

128

\section{Statistical analysis}

130 All statistical analyses were accomplished with $\mathrm{R}$ software 4.0.2. The Wilcoxon rank sum tests

131 were conducted for comparisons between two groups. Only a two-sided P-value $<0.05$ was

132 recognized as statistically significant. 
133 Results

134 Clinicopathological characteristics of LUADClinicopathological characteristics in the TCGA 135 database and GSE72094 are displayed in Table 1 and Table 2, respectively. In addition, complete 136 clinical information for each patient is listed in Table S1.

\section{Identification of DEmRNAs, DEmiRNAs and DEIncRNAs}

138

139

140

141

142

143

144

145

146

147

148

149

150

151

Figure1 presents our workflow for the bioinformatic analysis. Among the whole expression profiles, totally 1645 upregulated DEmRNAs, and 1344 downregulated DEmRNA were screened out (Fig. 2A,2B). Furthermore, we also identified 163 upregulated DElncRNA, 52 downregulated DElncRNA (Fig.2C,2D),111 upregulated DEmiRNA, and 87 downregulated DEmiRNA (Fig.,3B). Specifically, the top 20 significantly upregulated- and downregulated- miRNAs were shown in Fig.3A.

\section{Construct Nomogram of ceRNAs}

To investigate the endogenously competitive relationships between the lncRNA and mRNA, we constructed an intersected ceRNA network. Seven lncRNAs (H19, AC074117.1, FBXL19-AS1, MAGI2-AS3, SNHG3, PVT1 and SNHG1), 94mRNA, and 15miRNA were involved in the network (Table.S2). Hsa-miR-130b-3p and hsa-miR-29b-3p were the top 2 regulated miRNA in the ceRNA network (Fig. 4). In addition, the initial univariate Cox regression (Fig. S1) and Lasso regression analysis were further conducted to investigate the key biomarkers (Fig. 5B,5C). Seven 
152 survival-associated biomarkers were finally involved in a new multiple Cox regression analysis

153 (Fig. 5A). According to the result, all biomarkers belonged to protein-coding RNAs. Consequently,

154 the corresponding risk score was generated (Table 3): risk score $=[$ Exp CDC14A * $(-$

$1550.281888)]+(\operatorname{Exp} L O X L 2 * 0.114969)+(\operatorname{Exp}$ CCT6A *0.174244 $)+(\operatorname{Exp}$ E2F7 *0.187164 $)+(\operatorname{Exp}$

$156 \mathrm{GPR} 37 * 0.099737)+(\operatorname{Exp~H} 1 \mathrm{~F} 0 * 0.197590)+(\operatorname{Exp~SMOC1} * 0.068385)$.

157 As shown in Fig.5D, the prognostic score could be a promising indicator to distinguish the LUAD

158 patients according to the individual prognosis $(\mathrm{P}=5.701 \mathrm{e}-8)$. The area under ROC reflected the

159 predictive efficacy of the prognostic signature (AUC of 3-year survival:0.772) (Fig.5E). By the

160 GEO validation set, the AUC of gene signature at 3-year was 0.711 (Fig. S2A). Additionally, K-

$161 \mathrm{M}$ analysis further confirmed the difference between high- and low-risk groups $(\mathrm{P}=9.963 \mathrm{e}-05)$

162 (Fig. S2B). Eventually, we assessed the prognosis of patients with LUAD by a nomogram (Fig.

$1636 \mathrm{~A}$ ), and the accuracy of the nomogram was confirmed by the calibration curve (Fig. 6C). As

164 shown in Fig.7A, based on this prognostic model, the risk score in the group with fewer smoking

165 years $(\leqq 20)$ was lower than the group with more smoking years $(>20)(\mathrm{P}=0.025)$.

\section{Construct Nomogram of immune Cells}

167 By applying the CIBERSORT, we estimated the percentages of 22 immune cell types in LUAD

168 (Fig. S3B, S3C) (Chen et al. 2018). The violin plot indicated that 13 immune cell types showed a

169 significant difference in percentages of immune cells between tumor samples and healthy samples

170 by the Wilcoxon test (Fig. S3D). The correlation analysis of immune cells could be found in

171 Fig.S3A.Subsequently, after the analysis of univariate cox regression and lasso regression, seven 
172 immune cells were taken as prognostic biomarkers and eventually integrated into a new

173 multivariate model (Fig. 8A,8B,8C). The formula was presented as following (Table 4): risk score

$174=[\operatorname{Exp~CD8} *(-3.425649)]+($ Exp Tregs*9.175062 $)+[$ Exp Monocytes*(-5.318492) $]+($ Exp M1

$175 * 6.134551)+($ Exp Dendritic cells activated*5.539130 $)+($ Exp Mast cells activated *24.611110

$176)+($ Exp Eosinophils*37.351722). The K-M analysis results showed a significant difference in

177 overall survival between high- and low- risk groups ( $\mathrm{P}=3.342 \mathrm{e}-05)$ (Fig. 8D). The ROC curve

178 (AUC of 3-years: 0.766) demonstrated that the multivariate model was recognized as a fair

179 potential to monitor the prognostic efficacy(Fig. 8E). In the external validation set, the model also

180 carried out adequate predicted capacity. The AUC of immune cell signature was 0.738 at 3-years

181 (Fig. S2C).K-M curves reflected that the low-risk group had longer overall survival than the high-

182 risk group $(\mathrm{P}=2.922 \mathrm{e}-04)$ ( Fig. $\mathrm{S} 2 \mathrm{D})$. A predicted nomogram was also performed, and the

183 discrimination was conducted to test the calibration quality (Fig. 6B,6D). Similarly, we could also

184 found that the longer smoking history was related to the higher the risk scores based on the

predicted model of immune cells in smoking groups $(\mathrm{P}=0.0046)$ (Fig.7B). The correlations of key

prognostic biomarkers in the two models illustrate that the expression of E2F7 is positively related

to Macrohages M1 (R=0.4) (Fig.S4).

Besides, the Univariate and multivariate survival analyses revealed that risk scores in the two models were both independent prognostic factors (Fig.S5). 
192 193 194 195 196 197 198

As shown in Fig.9, CCT6A, H1F0 and E2F7 were positively related to RNAss and DNAss ( $\mathrm{R}=0.22$ to 0.53$)$. A significant negative association was detected between CDC14A and RNAss ( $\mathrm{R}=-0.25$, $\mathrm{P}=7.5 \mathrm{e}-8)$. Nevertheless, we did not find a significant correlation between LOXL2, GPR37 and SMOC1 with tumor stemness. In addition, H1F0 displayed a negative association with stromal scores, immune scores and estimate scores $(\mathrm{R}=-0.34$ to -0.27$)$. However, CDC14A showed a positive association with the microenvironment-related scores $(\mathrm{R}=0.31$ to 0.35$)$.

\section{Multiple databases validation}

To validate CDC14A and H1F0 expression in LUAD, we utilized multiple databases. Based on the result of UCLAN, GEPIA and TIMER, it could be concluded that H1F0 was highly expressed while CDC14A was weakly expressed in LUAD (Fig.S6). Furthermore, the CDC14A and H1F0 protein expression were obtained from The Human Protein Atlas data (HPA), indicating that H1F0 was high staining in tumor cells, while it was medium staining in normal tissues (Fig.S7A, S7B). The opposite trend was observed in CDC14A. Besides, the association of H1F0 and CDC14A with immune cells were validated in TISIDB. CDC14A expression was positively associated with Immature $B$ cell $(\mathrm{R}=0.345), \mathrm{NK}$ cell $(\mathrm{R}=0.308)$, Eosinophils $(\mathrm{R}=0.357)$, and Mast cell $(\mathrm{R}=0.332)$

(Fig.S8A-S8E). Controversially, H1F0 expression was negatively related to Eosinophil $(\mathrm{R}=-$ 0.34), Macrophage $(\mathrm{R}=-0.359), \operatorname{NKT}(\mathrm{R}=-0.323)$, Tem_CD8 $(\mathrm{R}=-0.323)$ and $\mathrm{Th} 1(\mathrm{R}=-0.407)$ (Fig.S8F-S8J).

\section{Differences in ssGSEA scores in TCGA/GEO cohort}


211 To further elucidate the immune status in risk- and low groups of ceRNA signatures, we performed

212 the ssGSEA algorithm to estimate the immune infiltrating between two groups (Fig.S9).Of note,

213 low-risk group were associated with higher scores of aDCs, B_cells, CD8+_T_cells,iDCs,

214 Mast_cells, Neutrophils, pDCs, T_helper_cells and TIL (Fig.10A,10B).Similarly, in the lower-risk

215 group, the scores of Check-point, HLA, T_cell_co-stimulation and Type_II_IFN_Reponse in still

216 higher in the low-risk group.Additionally, the differences above were confirmed by the GEO

217 cohort (Fig. 10C,10D). Discussion

218 With the reduced overall survival rate, lung adenocarcinoma is the most common pathological

219 type since most patients were diagnosed at an advanced stage. Currently, there is a general

220 consensus that both molecular and cellular characteristics play an imposing role in oncogenesis

221 and metastasis, and thus they were suggested as underlying prognostic signatures. However, the

222 integrative analysis of ceRNAs and microenvironments has yet to be fully explored. Previously,

223 there were several studies that explored potential biomarkers of diagnosis and prognosis in LUAD

224 via constructing the ceRNA model. Sui et al. constructed lncRNA-related network of LUAD with

22529 lncRNAs,24 miRNAs and 72mRNA (Sui et al. 2016). Then, they investigated the relevance of

226 lncRNA and clinical characteristics. Cao et al. established a ceRNA network consisting of 23

227 lncRNAs, 117mRNAs and 22miRNAs in lung cancer (Cao et al. 2020). Cao et al. also proposed a

228 6-IncRNA prognostic model. Li et al. constructed three ceRNA networks and a 32-ceRNA

229 prognostic signature ( $\mathrm{Li}$ et al. 2018). However, they did not further explore the correlation between

230 immune microenvironment and ceRNAs comprehensively. In the present study, we not only 
231 presented nomogram prediction models based on ceRNAs and immune cells, but also further

232 investigated the relationships between crucial ceRNAs and immune microenvironment.

233 GSE72094 dataset proved the feasibility of predicted equations.

Cigarette smoking is an established risk factor of lung cancer, which accounts for the leading cause of death worldwide (Ettinger et al. 2019).In recent years, the prevalence of LUAD has increased and smoking patients with LUAD constitute a certain proportion (Zhang et al. 2015).

237 Besides, we stratified TCGA patients with smoking history into three groups (smoking years $238>20$, smoking years $<=20$ and non-smoking ) and calculated risk scores in each sample via both predicted formulas, respectively. Not unexpectedly, both predicted formulas confirmed that the more extended smoking group (smoking years $>20$ ) have a higher risk than the shorter smoking group (smoking years $<=20$ ). Thus, it could be concluded that smoking was an adverse factor for prognosis in both models. Risk scores in the $>20$ years-smoking group were increased when compared with non-smoking groups in both models. However, there were no significant differences between $\leqslant$ the 20 years-smoking group and the non-smoking group. It was related to that the patients with less duration of smoking were more frequently female .E2F7, a member of EF transcription factors, was known as a transcriptional repressor that influences the prognosis in

247 a lot of tumors, such as breast cancer, endometrial carcinoma, gallbladder cancer, and colon cancer.

248 Previous studies have proved that miR-30a-5p prevents tumor migration and metastasis by targeting E2F7 in gallbladder cancer (Ye et al. 2018). Liu et al. have revealed that E2F7 was 
251 then led to tamoxifen resistance in breast cancer cells (Liu et al. 2018). Additionally, overexpressed

252 E2F7 could be suggested as a significant biomarker to identify the high-risk and low-risk groups

253 of patients with lung cancer, which is also in consistence with our report (Sun et al. 2018).

SMOC1 is a cancer-associated protein, identified as an extracellular glycoprotein of the SPARC-related modular calcium-binding protein family. SMOC1 was overexpressed in brain cancer, including oligodendrogliomas, astrocytomas, and glioblastomas (Brellier et al. 2011;

257

Fackler et al. 2011). However, till now, there is no enough evidence for the impact of SMOC1 in LUAD reported in the literature. Moreover, the current study may provide a study direction for researches of SMOC1 in the LUAD. Additionally, CCTA6 is reported to encode a molecular chaperone and play critical roles in damaged proteins repair, cytoskeletal organization, and the cell cycle (Van Hove et al. 2015; Yam et al. 2008). Ying et al. found that CCTA6 served as a SMAD2binding protein, which could inhibit the function of SMAD2, and promote metastasis (Ying et al. 2017).

CDC14A, which is of considerable significance to regulate the actin, was reported downregulated in many tumors and then reduced CDC14A was associated with the poor clinical outcomes (Chen et al. 2016). Our results indicated that CDC14A was a protective biomarker in LUAD $(\mathrm{HR}=0.75, \mathrm{P}=0.0066)$, which corresponded well with previous studies. Similarly, based on the correlation with stemness scores and immune socres, we could also draw inferences that CDC14A suppressed tumor progression.

As one of H1 histone genes, H1F0 (known as H1.0) is heterogeneous in many tumors (Di Liegro 
271 et al. 2018). Knockdown of H1.0 influenced the differentiation of embryonic stem cells (Terme et

272 al. 2011). Recent studies revealed that H1F0 were prognostic indicators in many cancers, such as

273 breast cancer, liver cancer, and kidney cancer (Torres et al. 2016).In the current study, the

274 relationships of H1F0 expression with stemness scores and Estamine scores suggested that H1F0

275 might promote LUAD.

276

$\mathrm{T}$ cells regulatory (Tregs) account for $5 \%-10 \%$ of peripheral CD4+ $\mathrm{T}$ cells in humans.

277 Increasing evidence demonstrated that the immunosuppression mediated by Tregs is one of the

278 essential mechanisms of immune evasion in tumors (Dunn et al. 2004; Shevach 2002; Zou 2005).

279 Mast cells are increased in several tumors, and their accumulation was associated with a low

280 survival rate in many cancers, such as pancreatic adenocarcinoma (Strouch et al. 2010) and

281 melanoma (Ribatti et al. 2003). Macrophages M1 was historically recognized as the

282 proinflammatory subgroup, expressing a series of chemokines and consequently play antitumor

283 roles (Hao et al. 2012). Controversially, our results suggested that M1 was highly expressed in

284 LUAD (Fig.8). Actually, heterogeneity of environment signals could influence TAM

285 development, and comprehensive nomenclatures of TAM have consequently been suggested

286 (Ostuni et al. 2015).

287 Additionally, our study found that there were obvious differences in immune status between

288 high- and low- risk groups in both TCGA and GEO cohorts. This indicated that decreased risk

289 scores of ceRNAs were related to the enhancement of antitumor immunity. Furthermore, we could

290 say that diminished immune function is responsible for poor prognosis in LUAD patients. 
291 However, there are inevitably some disadvantages in our study that should be taken into 292 consideration. Firstly, considering the limit data from the TCGA and GEO, it could result in large 293 analysis deviation and be verified by other large cohorts. Secondly, our follow-up experiment will 294 explore the molecular mechanisms of prognostic signatures in the present study.

\section{Conclusion}

296 To conclude, the present study firstly provided a combined analysis of ceRNA and immune cells 297 and then established nomograms to predict the prognosis in LUAD reliably. Based on the present 298 study result, we will carry out the biological experiments to validate key ceRNAs and mechanisms 299 of immune cells in the future.

\section{Acknowledgements}

The authors would like to thank TCGA, GEO, TIMER, GEPIA, HPA, UCLAN and TISIDB databases.

\section{Disclosure Statement}

304 No competing financial interests exist.

\section{Funding Information}

No funding was received for this article.

\section{References}

Barbie DA, Tamayo P, Boehm JS, Kim SY, Moody SE, Dunn IF, Schinzel AC, Sandy P, Meylan E, 
Scholl C, Fröhling S, Chan EM, Sos ML, Michel K, Mermel C, Silver SJ, Weir BA, Reiling JH, Sheng Q, Gupta PB, Wadlow RC, Le H, Hoersch S, Wittner BS, Ramaswamy S, Livingston DM, Sabatini DM, Meyerson M, Thomas RK, Lander ES, Mesirov JP, Root DE, Gilliland DG, Jacks T, and Hahn WC. 2009. Systematic RNA interference reveals that oncogenic KRASdriven cancers require TBK1. Nature 462:108-112. 10.1038/nature08460

Bartel DP. 2009. MicroRNAs: target recognition and regulatory functions. Cell 136:215-233. 10.1016/j.cell.2009.01.002

Bray F, Ferlay J, Soerjomataram I, Siegel RL, Torre LA, and Jemal A. 2018. Global cancer statistics 2018: GLOBOCAN estimates of incidence and mortality worldwide for 36 cancers in 185 countries. CA Cancer J Clin 68:394-424. 10.3322/caac.21492

Brellier F, Ruggiero S, Zwolanek D, Martina E, Hess D, Brown-Luedi M, Hartmann U, Koch M, Merlo A, Lino M, and Chiquet-Ehrismann R. 2011. SMOC1 is a tenascin-C interacting protein over-expressed in brain tumors. Matrix Biol 30:225-233. 10.1016/j.matbio.2011.02.001

Bruttel VS, and Wischhusen J. 2014. Cancer stem cell immunology: key to understanding tumorigenesis and tumor immune escape? Front Immunol 5:360. 10.3389/fimmu.2014.00360

Cao Q, Dong Z, Liu S, An G, Yan B, and Lei L. 2020. Construction of a metastasis-associated ceRNA network reveals a prognostic signature in lung cancer. Cancer Cell Int 20:208. 10.1186/s12935-020-01295-8

Chen B, Khodadoust MS, Liu CL, Newman AM, and Alizadeh AA. 2018. Profiling Tumor Infiltrating Immune Cells with CIBERSORT. Methods Mol Biol 1711:243-259. 10.1007/978-1-49397493-1_12

Chen NP, Uddin B, Voit R, and Schiebel E. 2016. Human phosphatase CDC14A is recruited to the cell leading edge to regulate cell migration and adhesion. Proc Natl Acad Sci U S A 113:990-995. 10.1073/pnas.1515605113

Chou CH, Shrestha S, Yang CD, Chang NW, Lin YL, Liao KW, Huang WC, Sun TH, Tu SJ, Lee WH, Chiew MY, Tai CS, Wei TY, Tsai TR, Huang HT, Wang CY, Wu HY, Ho SY, Chen PR, Chuang $\mathrm{CH}$, Hsieh PJ, Wu YS, Chen WL, Li MJ, Wu YC, Huang XY, Ng FL, Buddhakosai W, Huang PC, Lan KC, Huang CY, Weng SL, Cheng YN, Liang C, Hsu WL, and Huang HD. 2018. miRTarBase update 2018: a resource for experimentally validated microRNA-target interactions. Nucleic Acids Res 46:D296-d302. 10.1093/nar/gkx1067

Di Liegro CM, Schiera G, and Di Liegro I. 2018. H1.0 Linker Histone as an Epigenetic Regulator of Cell Proliferation and Differentiation. Genes (Basel) 9. 10.3390/genes9060310

Donnem T, Kilvaer TK, Andersen S, Richardsen E, Paulsen EE, Hald SM, AI-Saad S, Brustugun OT, Helland A, Lund-Iversen M, Solberg S, Gronberg BH, Wahl SG, Helgeland L, Fløtten O, Pohl M, Al-Shibli K, Sandanger TM, Pezzella F, Busund LT, and Bremnes RM. 2016. Strategies for clinical implementation of TNM-Immunoscore in resected nonsmall-cell lung cancer. Ann Oncol 27:225-232. 10.1093/annonc/mdv560

Dunn GP, Old $\amalg$, and Schreiber RD. 2004. The immunobiology of cancer immunosurveillance and immunoediting. Immunity 21:137-148. 10.1016/j.immuni.2004.07.017 
Ettinger DS, Wood DE, Aggarwal C, Aisner DL, Akerley W, Bauman JR, Bharat A, Bruno DS, Chang JY, Chirieac LR, D'Amico TA, Dilling TJ, Dobelbower M, Gettinger S, Govindan R, Gubens MA, Hennon M, Horn L, Lackner RP, Lanuti M, Leal TA, Lin J, Loo BW, Jr., Martins RG, Otterson GA, Patel SP, Reckamp KL, Riely GJ, Schild SE, Shapiro TA, Stevenson J, Swanson SJ, Tauer KW, Yang SC, Gregory K, and Hughes M. 2019. NCCN Guidelines Insights: NonSmall Cell Lung Cancer, Version 1.2020. J Natl Compr Canc Netw 17:1464-1472. 10.6004/jnccn.2019.0059

Fackler MJ, Umbricht CB, Williams D, Argani P, Cruz LA, Merino VF, Teo WW, Zhang Z, Huang P, Visvananthan K, Marks J, Ethier S, Gray JW, Wolff AC, Cope LM, and Sukumar S. 2011. Genome-wide methylation analysis identifies genes specific to breast cancer hormone receptor status and risk of recurrence. Cancer Res 71:6195-6207. 10.1158/00085472.can-11-1630

Hänzelmann S, Castelo R, and Guinney J. 2013. GSVA: gene set variation analysis for microarray and RNA-seq data. BMC Bioinformatics 14:7. 10.1186/1471-2105-14-7

Hao NB, Lü MH, Fan YH, Cao YL, Zhang ZR, and Yang SM. 2012. Macrophages in tumor microenvironments and the progression of tumors. Clin Dev Immunol 2012:948098. 10.1155/2012/948098

Imielinski M, Berger AH, Hammerman PS, Hernandez B, Pugh TJ, Hodis E, Cho J, Suh J, Capelletti M, Sivachenko A, Sougnez C, Auclair D, Lawrence MS, Stojanov P, Cibulskis K, Choi K, de Waal L, Sharifnia T, Brooks A, Greulich H, Banerji S, Zander T, Seidel D, Leenders F, Ansén S, Ludwig C, Engel-Riedel W, Stoelben E, Wolf J, Goparju C, Thompson K, Winckler W, Kwiatkowski D, Johnson BE, Jänne PA, Miller VA, Pao W, Travis WD, Pass HI, Gabriel SB, Lander ES, Thomas RK, Garraway LA, Getz G, and Meyerson M. 2012. Mapping the hallmarks of lung adenocarcinoma with massively parallel sequencing. Cell 150:11071120. 10.1016/j.cell.2012.08.029

Jeggari A, Marks DS, and Larsson E. 2012. miRcode: a map of putative microRNA target sites in the long non-coding transcriptome. Bioinformatics (Oxford, England) 28:2062-2063. 10.1093/bioinformatics/bts344

Kartha RV, and Subramanian S. 2014. Competing endogenous RNAs (ceRNAs): new entrants to the intricacies of gene regulation. Frontiers in genetics 5:8-8. 10.3389/fgene.2014.00008

Lavin Y, Kobayashi S, Leader A, Amir ED, Elefant N, Bigenwald C, Remark R, Sweeney R, Becker CD, Levine JH, Meinhof K, Chow A, Kim-Shulze S, Wolf A, Medaglia C, Li H, Rytlewski JA, Emerson RO, Solovyov A, Greenbaum BD, Sanders C, Vignali M, Beasley MB, Flores R, Gnjatic S, Pe'er D, Rahman A, Amit I, and Merad M. 2017. Innate Immune Landscape in Early Lung Adenocarcinoma by Paired Single-Cell Analyses. Cell 169:750-765.e717. 10.1016/j.cell.2017.04.014

Li J-H, Liu S, Zhou H, Qu L-H, and Yang J-H. 2014. starBase v2.0: decoding miRNA-ceRNA, miRNAncRNA and protein-RNA interaction networks from large-scale CLIP-Seq data. Nucleic Acids Res 42:D92-D97. 10.1093/nar/gkt1248

Liu J, Li X, Wang M, Xiao G, Yang G, Wang H, Li Y, Sun X, Qin S, Du N, Ren H, and Pang Y. 2018. A miR-26a/E2F7 feedback loop contributes to tamoxifen resistance in ER-positive breast 
391

392

393

394

395

396

397

398

399

400

401

402

403

404

405

406

407

408

409

410

411

412

413

414

415

416

417

418

419

420

421

422

423

424

425

426

427

428

429

430

431

cancer. Int J Oncol 53:1601-1612. 10.3892/ijo.2018.4492

Liu X, Niu X, and Qiu Z. 2020a. A Five-Gene Signature Based on Stromal/Immune Scores in the Tumor Microenvironment and Its Clinical Implications for Liver Cancer. DNA Cell Biol 39:1621-1638. 10.1089/dna.2020.5512

Liu Z, Wan Y, Qiu Y, Qi X, Yang M, Huang J, and Zhang Q. 2020b. Development and validation of a novel immune-related prognostic model in lung squamous cell carcinoma. Int J Med Sci 17:1393-1405. 10.7150/ijms.47301

Li X, Li B, Ran P, and Wang L. 2018. Identification of ceRNA network based on a RNA-seq shows prognostic IncRNA biomarkers in human lung adenocarcinoma. Oncol Lett 16:5697-5708. 10.3892/ol.2018.9336

Love MI, Huber W, and Anders S. 2014. Moderated estimation of fold change and dispersion for RNA-seq data with DESeq2. Genome Biol 15:550. 10.1186/s13059-014-0550-8

Malta TM, Sokolov A, Gentles AJ, Burzykowski T, Poisson L, Weinstein JN, Kamińska B, Huelsken J, Omberg L, Gevaert O, Colaprico A, Czerwińska P, Mazurek S, Mishra L, Heyn H, Krasnitz A, Godwin AK, Lazar AJ, Stuart JM, Hoadley KA, Laird PW, Noushmehr H, and Wiznerowicz M. 2018. Machine Learning Identifies Stemness Features Associated with Oncogenic Dedifferentiation. Cell 173:338-354.e315. 10.1016/j.cell.2018.03.034

Newman AM, Liu CL, Green MR, Gentles AJ, Feng W, Xu Y, Hoang CD, Diehn M, and Alizadeh AA. 2015. Robust enumeration of cell subsets from tissue expression profiles. Nat Methods 12:453-457. 10.1038/nmeth.3337

Ni SJ, Zhao LQ, Wang XF, Wu ZH, Hua RX, Wan CH, Zhang JY, Zhang XW, Huang MZ, Gan L, Sun HL, Dimri GP, and Guo WJ. 2018. CBX7 regulates stem cell-like properties of gastric cancer cells via p16 and AKT-NF-kB-miR-21 pathways. J Hematol Oncol 11:17. 10.1186/s13045018-0562-z

Ostuni R, Kratochvill F, Murray PJ, and Natoli G. 2015. Macrophages and cancer: from mechanisms to therapeutic implications. Trends Immunol 36:229-239. 10.1016/j.it.2015.02.004

Phelps M, Coss C, Wang H, and Cook M. 2016. Registered report: Coding-independent regulation of the tumor suppressor PTEN by competing endogenous mRNAs. Elife 5. 10.7554/eLife.12470

Qi X, Zhang DH, Wu N, Xiao JH, Wang X, and Ma W. 2015. ceRNA in cancer: possible functions and clinical implications. J Med Genet 52:710-718. 10.1136/jmedgenet-2015-103334

Ribatti D, Ennas MG, Vacca A, Ferreli F, Nico B, Orru S, and Sirigu P. 2003. Tumor vascularity and tryptase-positive mast cells correlate with a poor prognosis in melanoma. Eur J Clin Invest 33:420-425. 10.1046/j.1365-2362.2003.01152.x

Sanchez-Mejias A, and Tay Y. 2015. Competing endogenous RNA networks: tying the essential knots for cancer biology and therapeutics. J Hematol Oncol 8:30. 10.1186/s13045-0150129-1

Shannon P, Markiel A, Ozier O, Baliga NS, Wang JT, Ramage D, Amin N, Schwikowski B, and Ideker T. 2003. Cytoscape: a software environment for integrated models of biomolecular interaction networks. Genome Res 13:2498-2504. 10.1101/gr.1239303

Peer) reviewing PDF | (2020:10:53654:1:0:NEW 16 Jan 2021) 
Shevach EM. 2002. CD4+ CD25+ suppressor T cells: more questions than answers. Nat Rev Immunol 2:389-400. 10.1038/nri821

Shukla GC, Singh J, and Barik S. 2011. MicroRNAs: Processing, Maturation, Target Recognition and Regulatory Functions. Mol Cell Pharmacol 3:83-92.

Siegel RL, Miller KD, and Jemal A. 2018. Cancer statistics, 2018. CA Cancer J Clin 68:7-30. 10.3322/caac. 21442

Strouch MJ, Cheon EC, Salabat MR, Krantz SB, Gounaris E, Melstrom LG, Dangi-Garimella S, Wang E, Munshi HG, Khazaie K, and Bentrem DJ. 2010. Crosstalk between mast cells and pancreatic cancer cells contributes to pancreatic tumor progression. Clin Cancer Res 16:2257-2265. 10.1158/1078-0432.ccr-09-1230

Sui J, Li YH, Zhang YQ, Li CY, Shen X, Yao WZ, Peng H, Hong WW, Yin LH, Pu YP, and Liang GY. 2016. Integrated analysis of long non-coding RNA-associated ceRNA network reveals potential IncRNA biomarkers in human lung adenocarcinoma. Int J Oncol 49:2023-2036. 10.3892/ijo.2016.3716

Sun CC, Zhou Q, Hu W, Li SJ, Zhang F, Chen ZL, Li G, Bi ZY, Bi YY, Gong FY, Bo T, Yuan ZP, Hu WD, Zhan BT, Zhang Q, Tang QZ, and Li DJ. 2018. Transcriptional E2F1/2/5/8 as potential targets and transcriptional E2F3/6/7 as new biomarkers for the prognosis of human lung carcinoma. Aging (Albany NY) 10:973-987. 10.18632/aging.101441

Tay Y, Rinn J, and Pandolfi PP. 2014. The multilayered complexity of ceRNA crosstalk and competition. Nature 505:344-352. 10.1038/nature12986

Terme JM, Sesé B, Millán-Ariño L, Mayor R, Izpisúa Belmonte JC, Barrero MJ, and Jordan A. 2011. Histone $\mathrm{H} 1$ variants are differentially expressed and incorporated into chromatin during differentiation and reprogramming to pluripotency. J Biol Chem 286:35347-35357. 10.1074/jbc.M111.281923

Torres CM, Biran A, Burney MJ, Patel H, Henser-Brownhill T, Cohen AS, Li Y, Ben-Hamo R, Nye E, Spencer-Dene B, Chakravarty P, Efroni S, Matthews N, Misteli T, Meshorer E, and Scaffidi P. 2016. The linker histone $\mathrm{H} 1.0$ generates epigenetic and functional intratumor heterogeneity. Science 353. 10.1126/science.aaf1644

Van Hove I, Verslegers M, Hu TT, Carden M, Arckens L, and Moons L. 2015. A proteomic approach to understand MMP-3-driven developmental processes in the postnatal cerebellum: Chaperonin CCT6A and MAP kinase as contributing factors. Dev Neurobiol 75:1033-1048. 10.1002/dneu.22272

Yam AY, Xia Y, Lin HT, Burlingame A, Gerstein M, and Frydman J. 2008. Defining the TRiC/CCT interactome links chaperonin function to stabilization of newly made proteins with complex topologies. Nat Struct Mol Biol 15:1255-1262. 10.1038/nsmb.1515

Ye YY, Mei JW, Xiang SS, Li HF, Ma Q, Song XL, Wang Z, Zhang YC, Liu YC, Jin YP, Hu YP, Jiang L, Liu FT, Zhang YJ, Hao YJ, and Liu YB. 2018. MicroRNA-30a-5p inhibits gallbladder cancer cell proliferation, migration and metastasis by targeting E2F7. Cell Death Dis 9:410. 10.1038/s41419-018-0444-x

Ying Z, Tian H, Li Y, Lian R, Li W, Wu S, Zhang HZ, Wu J, Liu L, Song J, Guan H, Cai J, Zhu X, Li J, and Li M. 2017. CCT6A suppresses SMAD2 and promotes prometastatic TGF- $\beta$ signaling. J Clin 
473

474

475

476

477

478

479

480

481

482

483

484

Invest 127:1725-1740. 10.1172/jci90439

Yoshihara K, Shahmoradgoli M, Martínez E, Vegesna R, Kim H, Torres-Garcia W, Treviño V, Shen H, Laird PW, Levine DA, Carter SL, Getz G, Stemke-Hale K, Mills GB, and Verhaak RG. 2013. Inferring tumour purity and stromal and immune cell admixture from expression data. Nat Commun 4:2612. 10.1038/ncomms3612

Zhang L, Li M, Wu N, and Chen Y. 2015. Time Trends in Epidemiologic Characteristics and Imaging Features of Lung Adenocarcinoma: A Population Study of 21,113 Cases in China. PLoS One 10:e0136727. 10.1371/journal.pone.0136727

Zou W. 2005. Immunosuppressive networks in the tumour environment and their therapeutic relevance. Nat Rev Cancer 5:263-274. 10.1038/nrc1586 


\section{Figure 1}

Figure 1 The workflow for bioinformatic analysis

\section{Transcriptomic profiles of 535 samples with LUAD} and 59 normal samples(TCGA)

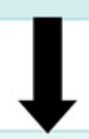

Differentially expressed gene analysis (DEmRNA, DEmiRNA, DEIncRNA)

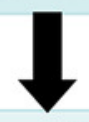

Construction of LUAD-specific ceRNA network

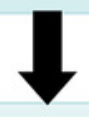

Construction of the predicted model based on hub ceRNAs

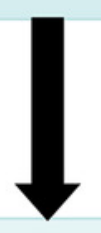

Comparasion of ssGSEA scores in highand low-risk group

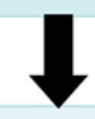

External validation the prognostic models via independent dataset (GSE72094)

Construction of the predicted model based on hub immune cells

Estimate the proportion of immune cell in tumor samples and normal samples

Identification of survival-associated immune cells

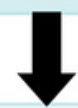

I

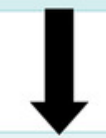

correlation analysis of hub genes with immune cells/immune scores

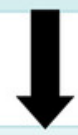

Comparasion of risk scores in $\leqq 20$ years-smoking,

$>20$ years-smoking and non-smoking groups 


\section{Figure 2}

Figure 2 Differentially expressed mRNAs and IncRNA.

Heatmap (A) and volcano plot (B) of DEmRNA.Heatmap (C) and volcano plot (D) of DEIncRNA. Red dots and green dots represent up -regulated, down-regulated differentially expressed genes, respectively. DEmRNA and DEIncRNA represent differentially expressed mRNA and IncRNA, respectively.

A

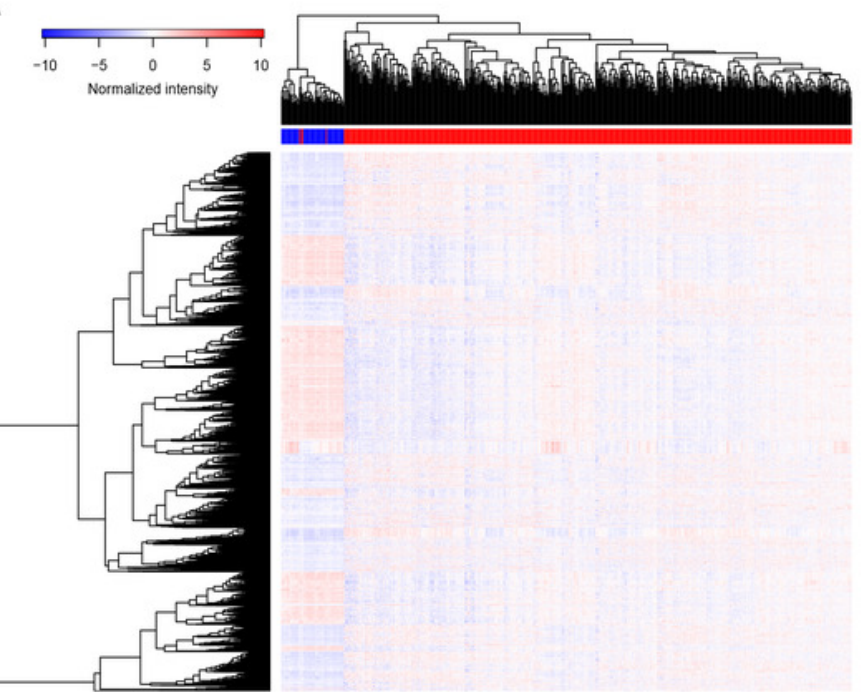

C
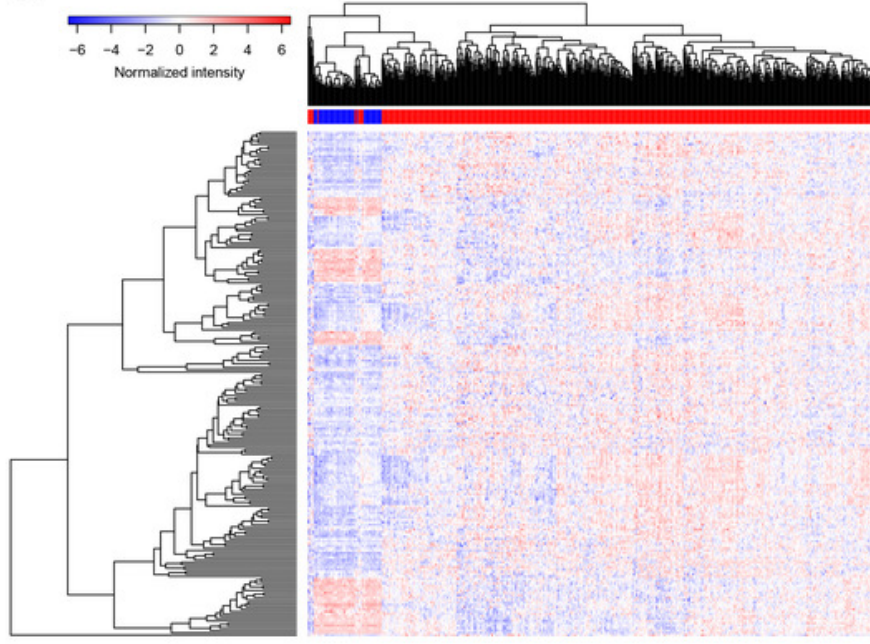

B

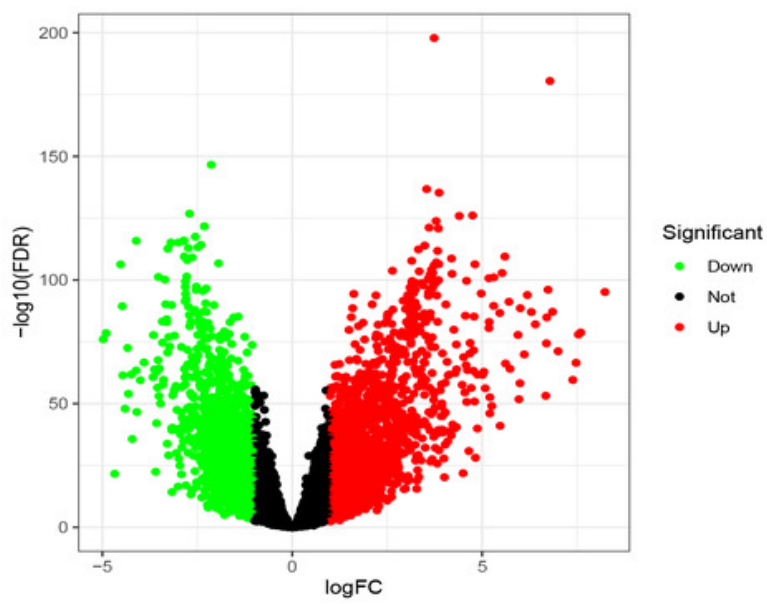

D

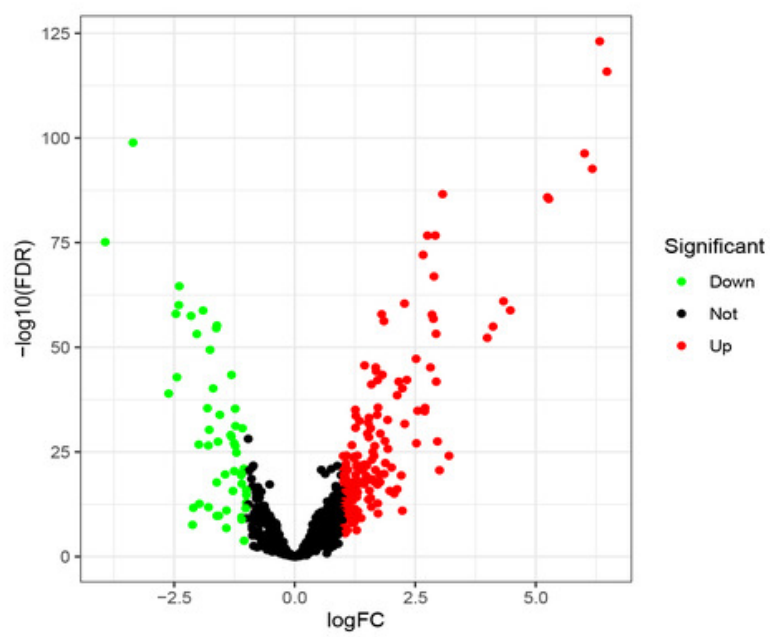




\section{Figure 3}

Figure 3 Differentially expressed miRNAs.

Heatmaps of the top 20 differentially upregulated and the top 20 differentially downregulated miRNAs (A).Volcano plot (B) of DEmiRNAs. Red dots and green dots represent up-regulated, down-regulated differentially expressed genes, respectively.DEmiRNAs, differentially expressed miRNAs.

A

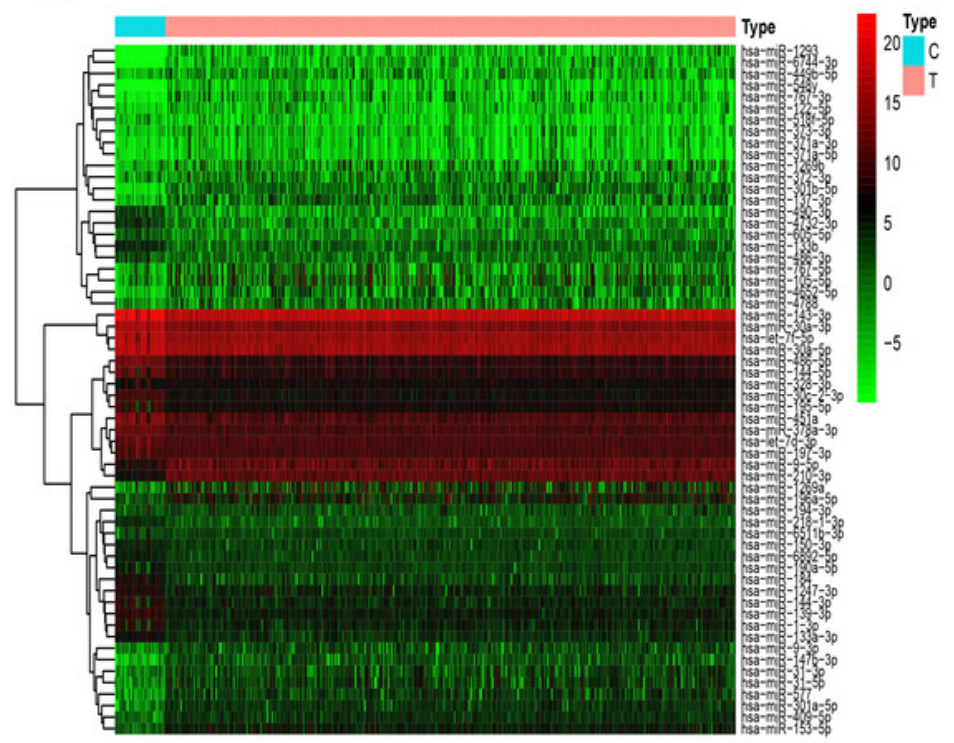

B

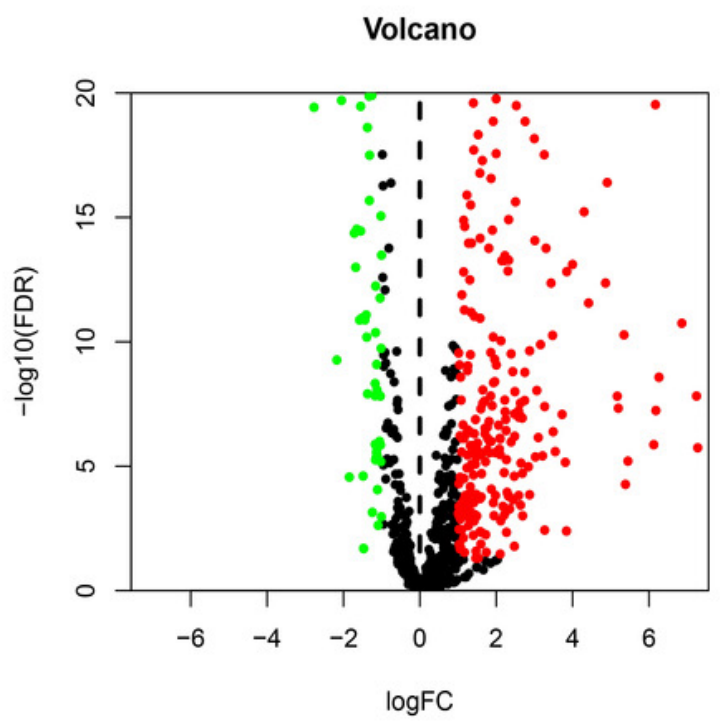




\section{Figure 4}

Figure 4 Construction of the ceRNA network in LUAD.

Red circles, purple squares, green diamonds represent miRNA, mRNA and IncRNA, respectively. Node sides of miRNA indicate the number of junction nodes. The greater the node sizes, the more junction nodes. 


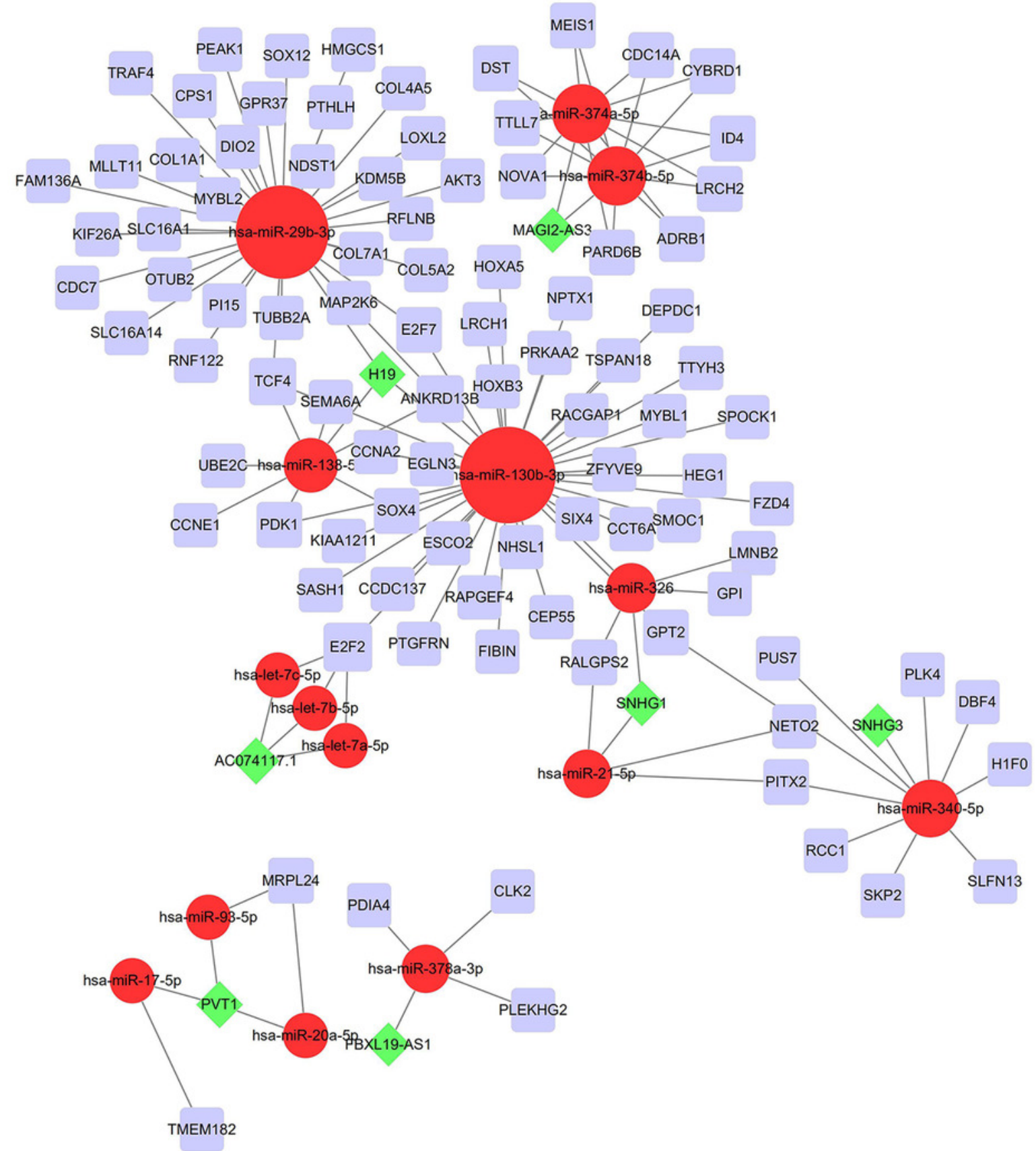


Figure 5

Figure 5 Construction of the prognostic model involving the key RNAs of the ceRNA network in LUAD.

(A), multivariate Cox regression. (B), (C) lasso regression. (D) the Kaplan-Meier survival

curve. (E) the ROC curve. ROC, the Receiver Operating Characteristic. LUAD, lung adenocarcinoma. 
A

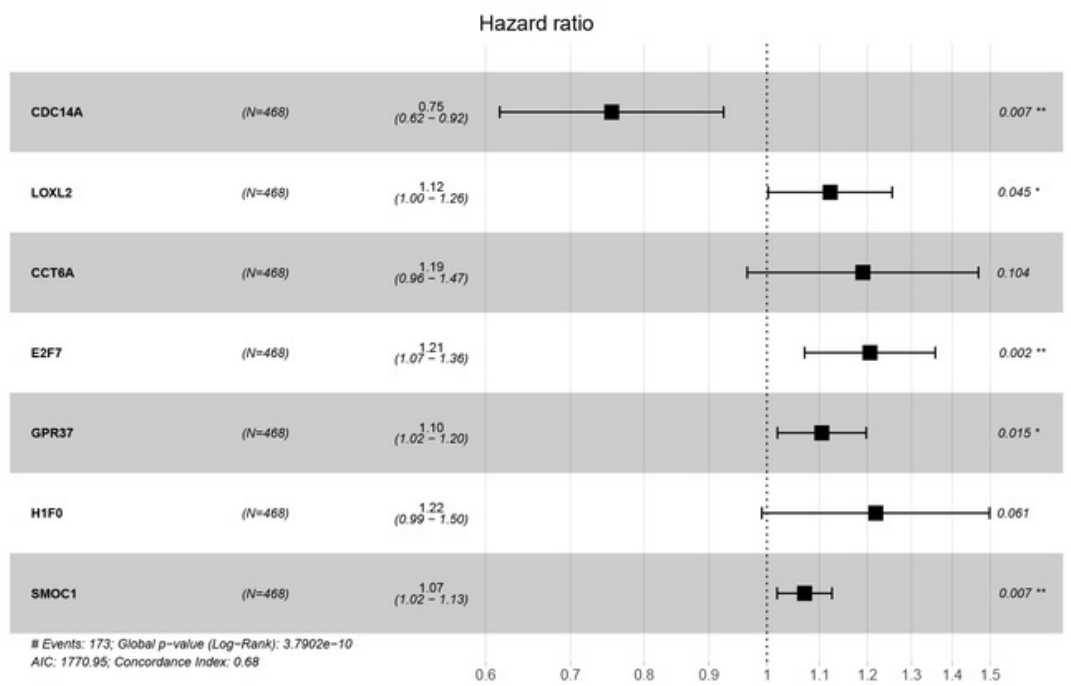

B
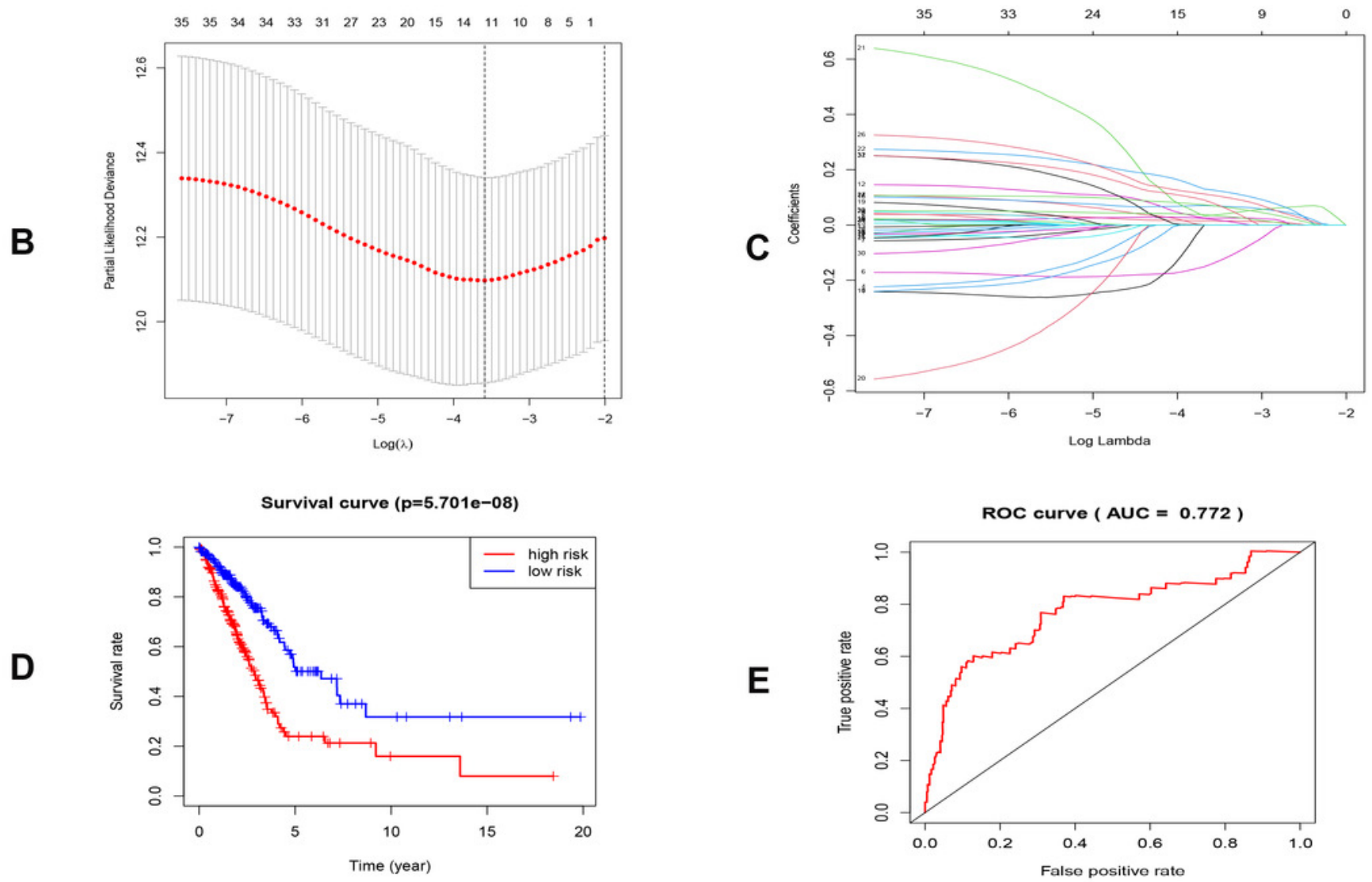
Figure 6

Figure 6 Nomograms based on key ceRNAs and immune cells.

Nomograms predicting 1-,2- and 3-year overall survival of ceRNAs signatures (A) and immune cells signatures (B).Calibration curves of the nomogram at the 3-year of ceRNAs (C) signatures and immune cells signatures (D).

A

Points

CDC14A

LOXL2

ССт6A

E2F7

GPR37

H1FO

SMOC1

Total Points

1-year survival

2-year survival

3-year survival

C

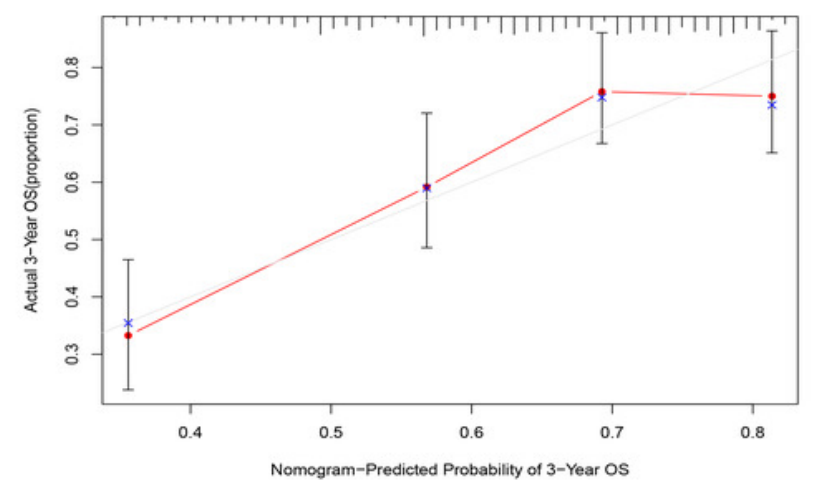

B

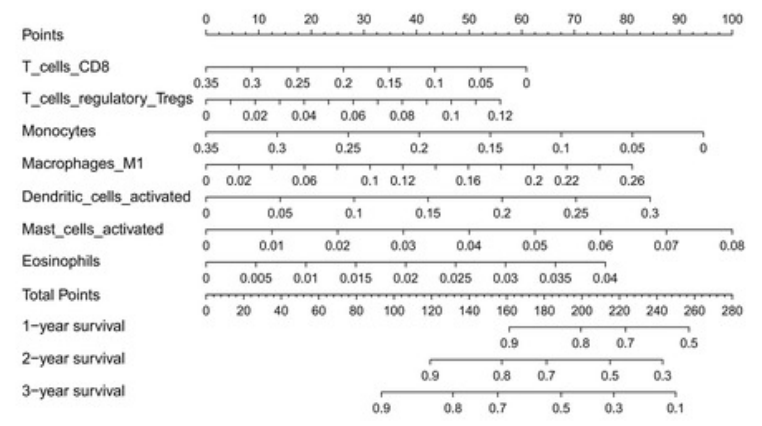

D

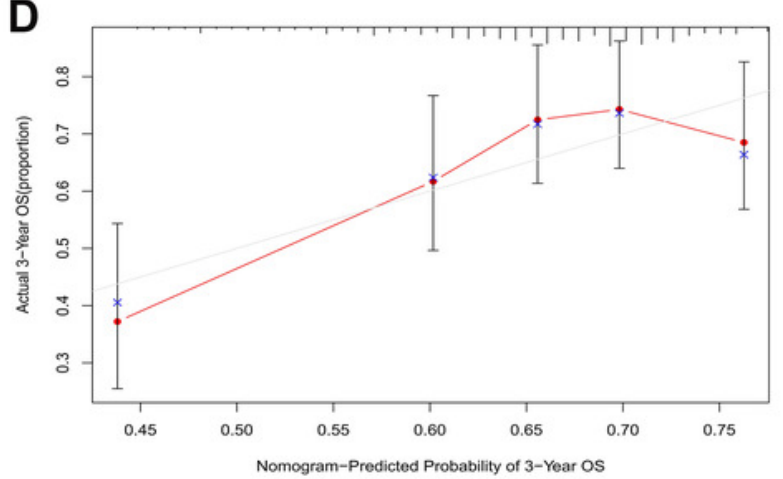


Figure 7

Figure7 Differences in risk scores between $>20$-smoking years, $\leq 20$-smoking years and non-smoking based on ceRNAs (A) and immune cells models (B).

A

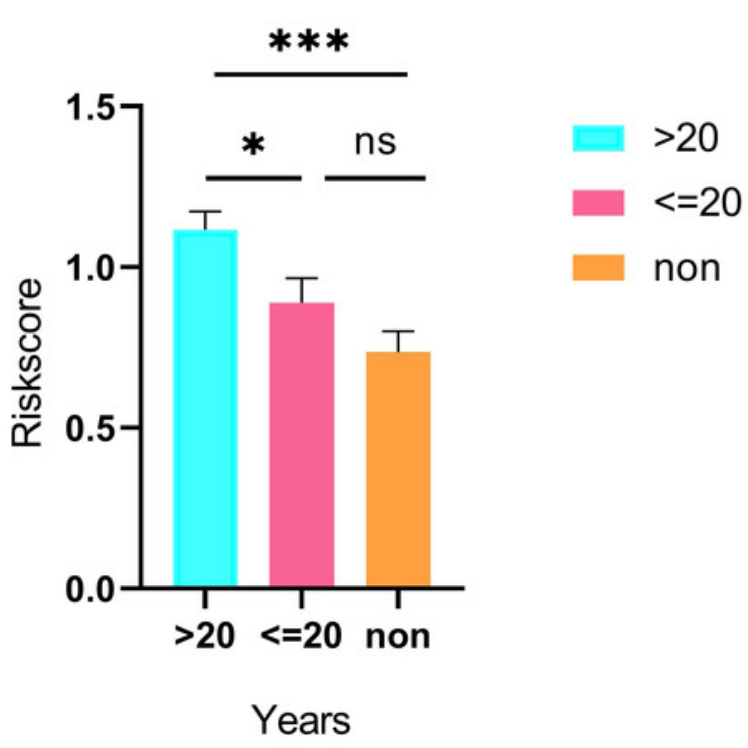

B

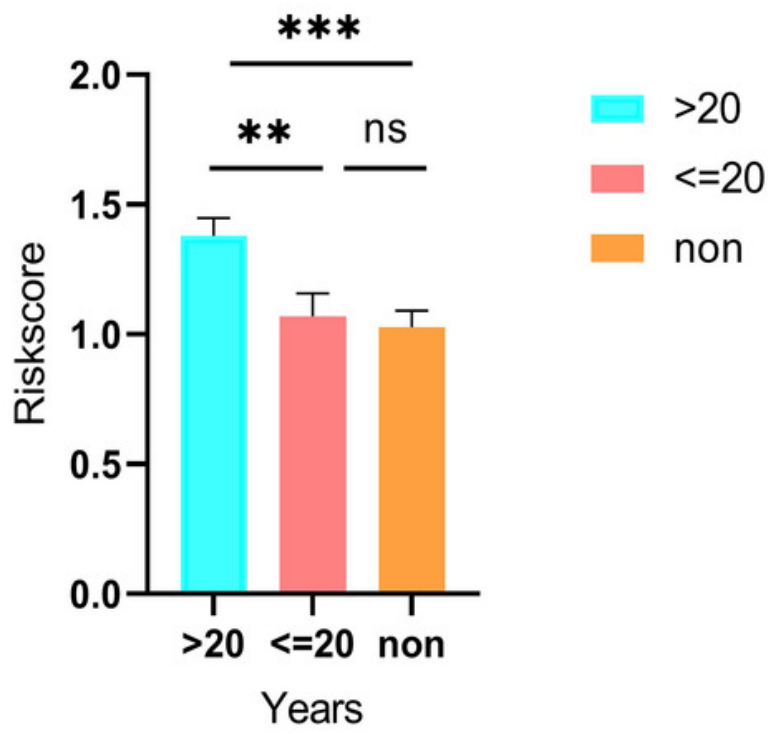


Figure 8

Figure 8 Construction of the prognostic model based on key immune cells in LUAD

(A), multivariate Cox regression. (B), (C) lasso regression. (D) the Kaplan-Meier survival curve. (E) the ROC curve. ROC, the Receiver Operating Characteristic. LUAD, lung adenocarcinoma 


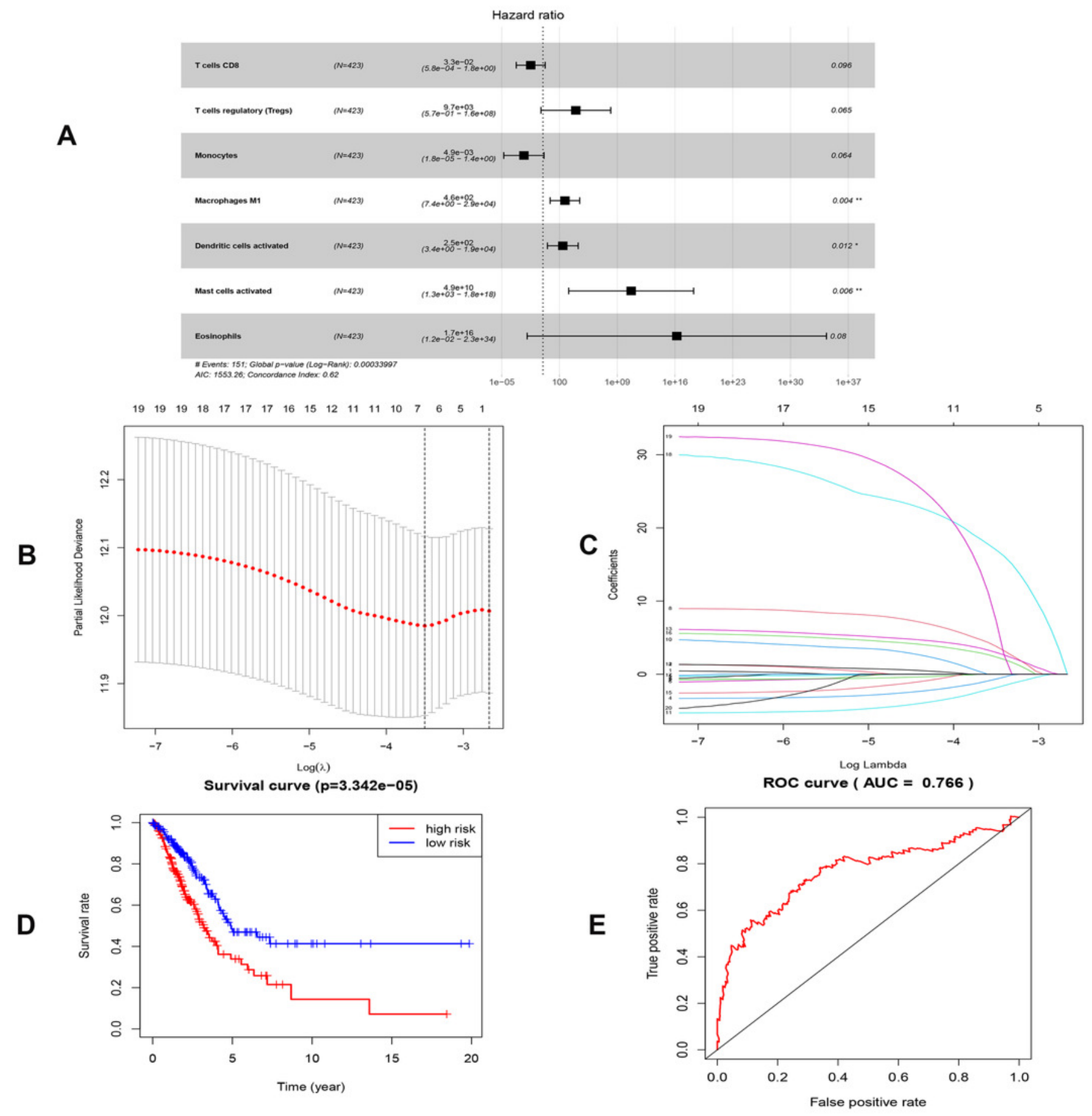




\section{Figure 9}

Figure 9 Correlations between crucial ceRNAs expression and stem cell index, crucial ceRNAs expression and Stromal/Immune/ESTIMATE score in LUAD

RNAss and DNAss represent RNA-based stemness score, DNA-based stemness score, respectively. The sum of Stromal score and Immune score is ESTIMATE Score.

Cancer: LUAD
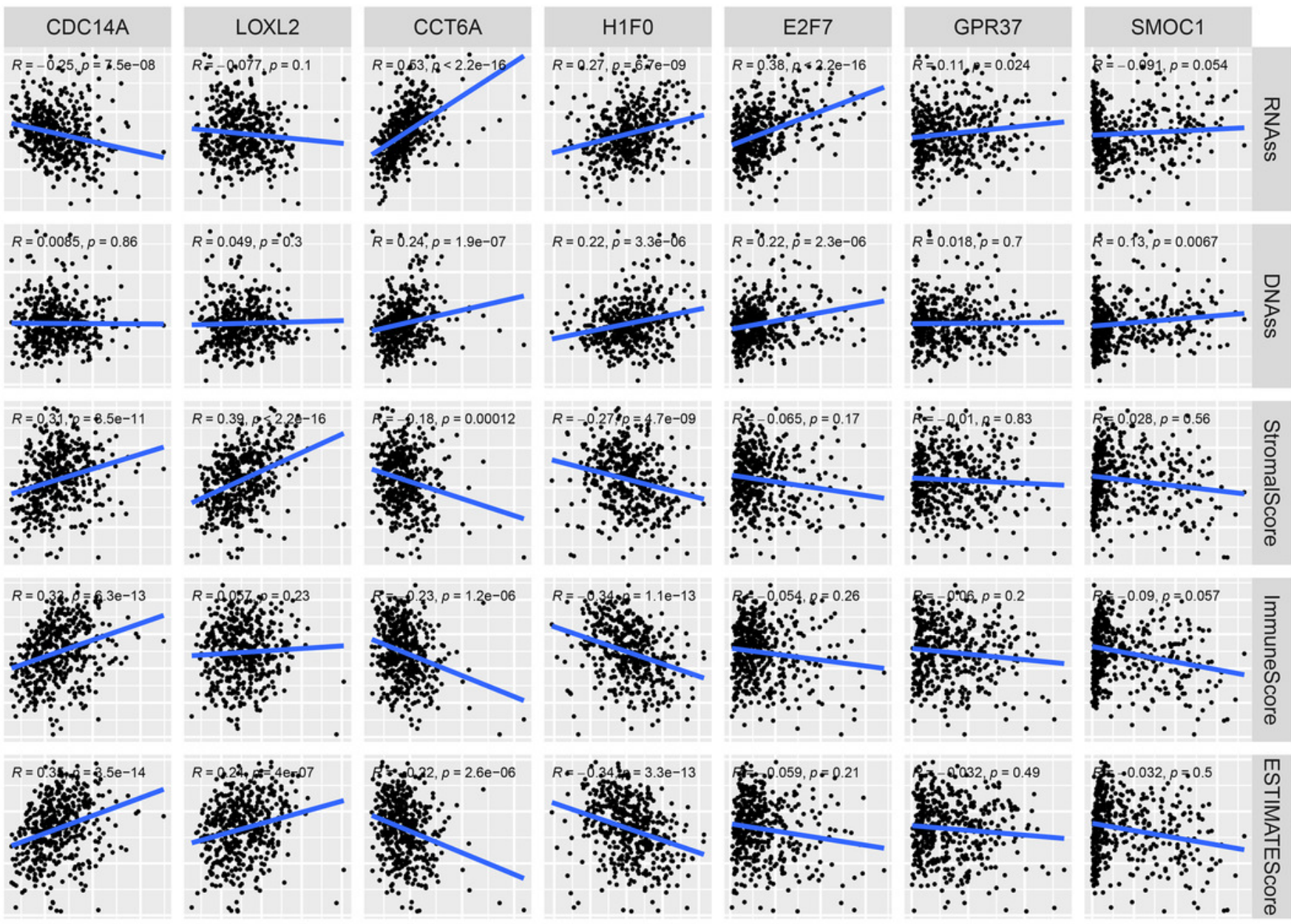

Gene expression 
Figure 10

Figure 10 Differences of ssGSEA scores in high- and low- risk groups in TCGA (A)(B) cohort and GEO (C)(D) cohort

(A),(C) refer to the difference in immune cells.(B),(D) refer to the difference in immuneassociated functions $* p<0.05, * * p<0.01$, and ${ }^{* * *} p<0.001$

A

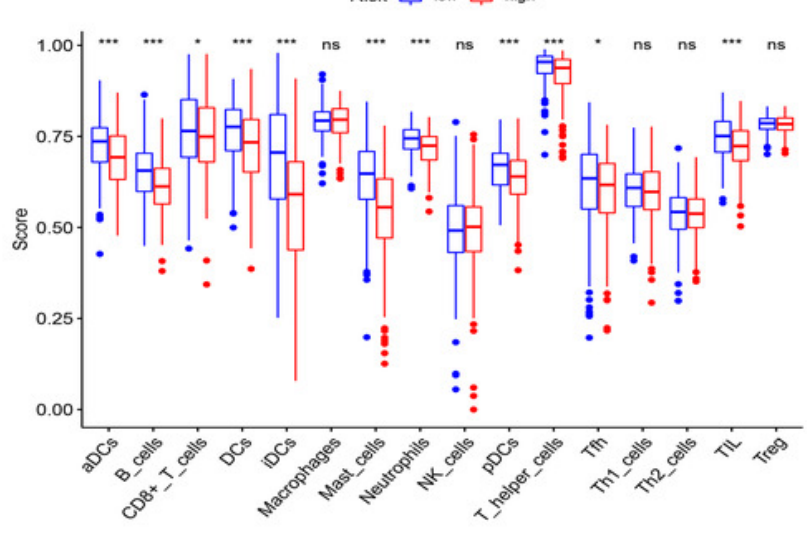

C
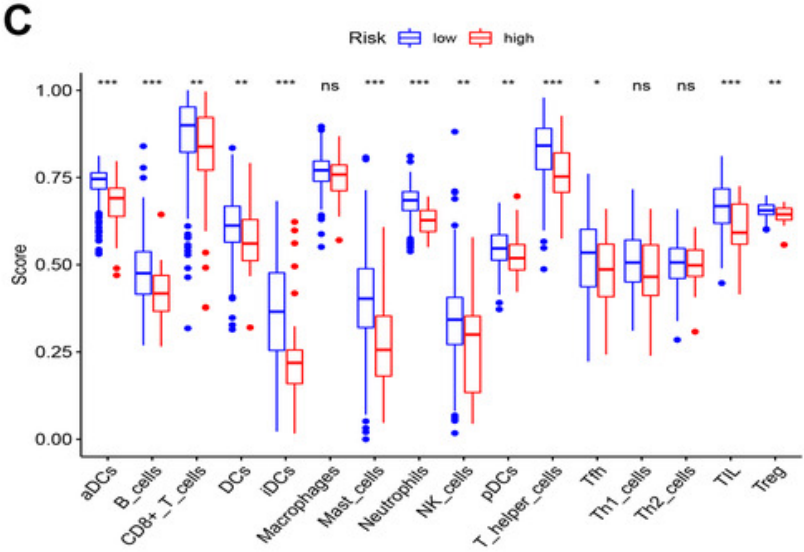

B

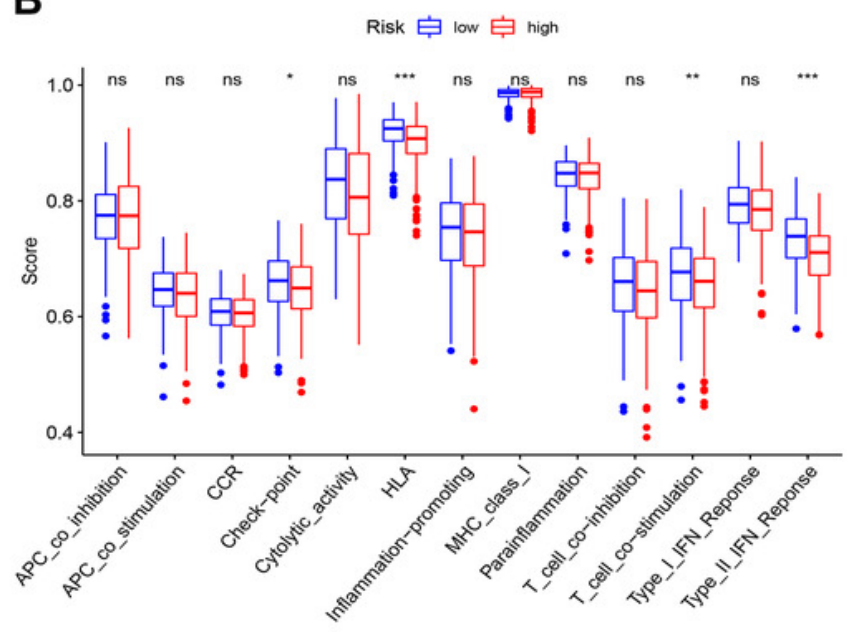

D

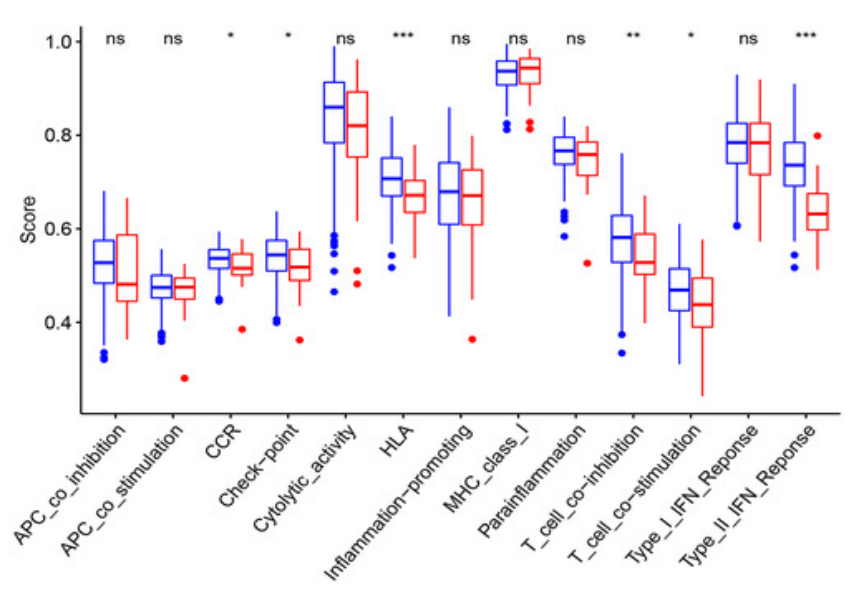




\section{Table 1 (on next page)}

Clinicopathological characteristics baseline in LUAD in TCGA 
1 Table 1

\section{Clinicopathological characteristics baseline in LUAD in TCGA}

Characteristics

Total

$\begin{array}{rr}\text { number } & (\%) \\ 522 & 100 \%\end{array}$

Age

$$
>65
$$

$262 \quad 50.2 \%$

$<=65$

$241 \quad 46.2 \%$

unkown

$19 \quad 3.6 \%$

Gender

Female

$280 \quad 53.6 \%$

Male

$242 \quad 46.4 \%$

Vital status

dead

$188 \quad 36.0 \%$

alive

$334 \quad 64.0 \%$

Clinical stage

T stage

I

II

III

IV

unkown

T1

T2

T3

T4

unkown

M stage

$\mathrm{N}$ stage

M0

M1

unkown

N0

N1

N2

N3

unkown

Smoking status

$<=20$

$>20$
$279 \quad 53.4 \%$

$114 \quad 21.8 \%$

$85 \quad 16.3 \%$

$26 \quad 5.0 \%$

$8 \quad 1.5 \%$

$172 \quad 33.0 \%$

$281 \quad 53.8 \%$

$47 \quad 9.0 \%$

$19 \quad 3.6 \%$

$3 \quad 0.6 \%$

$353 \quad 67.6 \%$

$25 \quad 4.8 \%$

$144 \quad 27.6 \%$

$335 \quad 64.2 \%$

$98 \quad 18.8 \%$

$75 \quad 14.4 \%$

$20.4 \%$

$12 \quad 0.2 \%$

$45 \quad 8.6 \%$

$139 \quad 26.6 \%$ 
unkown

$338 \quad 64.80 \%$

2 LUAD, lung adenocarcinoma 
Table 2 (on next page)

Clinicopathological characteristics baseline in LUAD in GSE72094 
1 Table 2

\section{Clinicopathological characteristics baseline in LUAD in GSE72094}

Characteristics

Total

$\begin{array}{rr}\text { number } & (\%) \\ 442 & 100 \% \\ & \\ 294 & 50.2 \% \\ 127 & 46.2 \% \\ 21 & 3.6 \%\end{array}$

Gender

$>65$
$<=65$
unkown

Female

280

$53.6 \%$

Male

242

$46.4 \%$

Smoking status

never

335

$75.8 \%$

unkown

33

$7.5 \%$

74

$16.7 \%$

Stage

\section{I}

II

III

IV

unkown

Vital Status
Alive

Dead

unkown
$265 \quad 60.0 \%$

$69 \quad 15.6 \%$

$63 \quad 14.3 \%$

$17 \quad 3.9 \%$

$28 \quad 6.3 \%$

$298 \quad 67.4 \%$

$122 \quad 27.6 \%$

$22 \quad 5.0 \%$

2 


\section{Table 3(on next page)}

Multivariate Cox proportional hazards regression model based on ceRNAs in LUAD 
1 Table 3 Multivariate Cox proportional hazards regression model based on ceRNAs in 2 LUAD.

\begin{tabular}{ccccc}
\hline Genes & Coef & HR & $95 \%$ CI & P value \\
\hline CDC14A & -0.282 & 0.754 & $(0.616-0.924)$ & 0.007 \\
LOXL2 & 0.115 & 1.122 & $(1.002-1.256)$ & 0.045 \\
CCT6A & 0.174 & 1.190 & $(0.965-1.468)$ & 0.104 \\
E2F7 & 0.187 & 1.206 & $(1.071-1.358)$ & 0.002 \\
GPR37 & 0.100 & 1.105 & $(1.019-1.198)$ & 0.015 \\
H1F0 & 0.198 & 1.218 & $(0.991-1.498)$ & 0.061 \\
SMOC1 & 0.068 & 1.071 & $(1.019-1.125)$ & 0.007 \\
\hline
\end{tabular}

3 LUAD lung adenocarcinoma, HR hazrd ratio, CI confidence interval.

4 
Table 4 (on next page)

Multivariate Cox proportional hazards regression model based on Immune cells in LUAD. 
1 Table 4 Multivariate Cox proportional hazards regression model based on Immune cells 2 in LUAD.

\begin{tabular}{ccccc}
\hline Immune cells & Coef & HR & $95 \%$ CI & P value \\
\hline T cells CD8 & -3.426 & 0.033 & $(5.767 \mathrm{e}-04-1.835)$ & 0.096 \\
T cells regulatory (Tregs) & 9.175 & 9653.370 & $(0.571-1.631 \mathrm{e}+08)$ & 0.065 \\
Monocytes & -5.318 & 0.005 & $(1.778 \mathrm{e}-05-1.351)$ & 0.064 \\
Macrophages M1 & 6.135 & 461.532 & $(7.447-2.860 \mathrm{e}+04)$ & 0.004 \\
Dendritic cells activated & 5.539 & 254.456 & $(3.446-1.879 \mathrm{e}+04)$ & 0.012 \\
Mast cells activated & 24.611 & $4.881 \mathrm{e}+10$ & $(1.320 \mathrm{e}+03-1.805 \mathrm{e}+18)$ & 0.006 \\
& & $1.66589 \mathrm{E}+$ & $(0.012-2.282 \mathrm{e}+24)$ & 0.080 \\
Eosinophils & 37.352 & 16 & &
\end{tabular}

3 LUAD lung adenocarcinoma, Coef correlation coefficients, HR hazrd ratio,CI confidence interval. 